\title{
Dynamic Buffer Capacity in Acid-Base Systems
}

\author{
Anna M. Michałowska-Kaczmarczyk ${ }^{1}$. \\ Tadeusz Michałowski ${ }^{2}$
}

Received: 2 October 2014/ Accepted: 29 December 2014/Published online: 16 June 2015

(C) The Author(s) 2015. This article is published with open access at Springerlink.com

\begin{abstract}
The generalized concept of 'dynamic' buffer capacity $\beta_{V}$ is related to electrolytic systems of different complexity where acid-base equilibria are involved. The resulting formulas are presented in a uniform and consistent form. The detailed calculations are related to two Britton-Robinson buffers, taken as examples.
\end{abstract}

Keywords Acid-base equilibria $\cdot$ Buffer capacity $\cdot$ Titration

\section{Introduction}

Buffer solutions are commonly applied in many branches of classical and instrumental analyses [1, 2], e.g. in capillary electrophoresis, CE [3-5], and polarography [6]. The effectiveness of a buffer at a given $\mathrm{pH}$ is governed mainly by its buffer capacity $(\beta)$, defined primarily by Van Slyke [7]. The $\beta$-concept refers usually to electrolytic systems where only one proton/acceptor pair exists. A more general (and elegant) formula for $\beta$ was provided by Hesse and Olin [8] for the system containing a $n$-protic weak acid $\mathrm{H}_{n} \mathrm{~L}$ together with strong acid, $\mathrm{HB}$, and strong base, $\mathrm{MOH}$; it was an extension of the $\beta$-concept from [9]. The formula for $\beta$ found in the literature is usually referred to the 'static' case, based on an assumption that total concentration of the species forming a buffering system is unchanged. The dilution effects, resulting from addition of finite volume of an acid or base to such dynamic systems during titrations, was considered in the papers [2,10], where finite changes $(\Delta \mathrm{pH})$ in $\mathrm{pH}$, affected by addition of the strong acid or base, were closely related to the formulas for the acid-base titration curves. The $\Delta \mathrm{pH}$ values, called

Tadeusz Michałowski

michalot@o2.pl

1 Department of Oncology, The University Hospital in Cracow, Cracow, Poland

2 Faculty of Chemical Engineering and Technology, Cracow University of Technology, Warszawska 24, 31-155 Cracow, Poland 
'windows', were considered later [11] for a mixture of monoprotic acids titrated with $\mathrm{MOH}$; the dynamic version of this concept was presented first in [10].

Buffering action is involved with mixing of two (usually aqueous) solutions. The mixing can be performed according to the titrimetric mode. In the present paper, the formula for dynamic buffer capacity, $\beta_{V}=\left|\frac{\mathrm{d} c}{\mathrm{dpH}}\right|$ related to the systems where $V_{0} \mathrm{~mL}$ of the solution being titrated (titrand, D) of different complexity, with concentrations $\left[\mathrm{mol} \cdot \mathrm{L}^{-1}\right.$ ] of component(s) denoted by $C_{0}$ or $C_{0 k}$, is titrated with $V \mathrm{~mL}$ of $C \mathrm{~mol} \cdot \mathrm{L}^{-1}$ solution of: $\mathrm{MOH}$ (e.g. $\mathrm{NaOH}), \mathrm{HB}$ (e.g. $\mathrm{HCl}$ ), or a weak polyprotic acid $\mathrm{H}_{n} \mathrm{~L}$ or its salt of $\mathrm{M}_{m} \mathrm{H}_{n-m} \mathrm{~L}(m=1, \ldots, n)$, or $\mathrm{H}_{n+m} \mathrm{LB}_{m}$ type as a reagent in titrant $(\mathrm{T})$ are considered. This way, the $\mathrm{D}+\mathrm{T}$ mixture of volume $V_{0}+V \mathrm{~mL}$, is obtained, if the assumption of additivity of the volumes is valid. It is assumed that, at any stage of the titration, $\mathrm{D}+\mathrm{T}$ is a monophase system where only acid-base reactions occur. The formation function $\bar{n}=\bar{n}(p H)$ $[12,13]$ was incorporated, as a very useful concept, into formulas for acid-base titration curves, obtained on the basis of charge and concentration balances, referred to polyprotic acids.

\section{Definition of Dynamic Buffer Capacity}

In this work, the buffer capacity is defined as follows:

$$
\beta_{V}=\left|\frac{\mathrm{d} c}{\mathrm{dpH}}\right|
$$

where

$$
c=C \cdot \frac{V}{V_{0}+V} \equiv C-\frac{C \cdot V_{0}}{V_{0}+V}
$$

denotes the current concentration of a reagent $\mathrm{R}$ in a $\mathrm{D}+\mathrm{T}$ mixture obtained after addition of $V \mathrm{~mL}$ of $C \mathrm{~mol} \cdot \mathrm{L}^{-1}$ solution of the reagent $\mathrm{R}$ (considered as titrant, $\mathrm{T}$ ) into $V_{0} \mathrm{~mL}$ of a solution named as titrand (D). From Eqs. 1 and 2 we have:

$$
\beta_{V}=\left|\frac{\mathrm{d} c}{\mathrm{~d} V} \cdot \frac{\mathrm{d} V}{\mathrm{dpH}}\right|=\frac{C \cdot V_{0}}{\left(V_{0}+V\right)^{2}} \cdot\left|\frac{\mathrm{d} V}{\mathrm{dpH}}\right|
$$

The buffer capacity $\beta_{V}$ is an intensive property, expressed in terms of molar concentrations, i.e., intensive variable. The expressions for $\frac{\mathrm{d} V}{\mathrm{dpH}}$ in Eq. 3 will be formulated below.

\section{Formulation of Dynamic Buffer Capacity}

Some particular systems can be distinguished. For the sake of simplicity in notation, the charges of particular species $X_{i}^{z_{i}}$ will can be omitted when put in square brackets, expressing molar concentration $\left[X_{i}\right]$.

System 1A: $V \mathrm{~mL}$ of $\mathrm{MOH}\left(C, \mathrm{~mol} \cdot \mathrm{L}^{-1}\right)$ is added, as reagent $\mathrm{R}$, into $V_{0} \mathrm{~mL}$ of $\mathrm{K}_{m-}$ $\mathrm{H}_{n-m} \mathrm{~L}\left(C_{0}, \mathrm{~mol} \cdot \mathrm{L}^{-1}\right)$. The concentration balances are as follows: 


$$
[\mathrm{M}]=C V /\left(V_{0}+V\right) ;[\mathrm{K}]=m \cdot C_{0} V_{0} /\left(V_{0}+V\right) ; \sum_{i=0}^{q}[\mathrm{H} \cdot \mathrm{L}]=\frac{C_{0} V_{0}}{V_{0}+V}
$$

Denoting:

$$
\begin{gathered}
{\left[\mathrm{H}_{i} \mathrm{~L}\right]=K_{i}^{\mathrm{H}} \cdot[\mathrm{H}]^{i} \cdot[\mathrm{L}] ; b_{i}=K_{i}^{\mathrm{H}} \cdot[\mathrm{H}]^{i} ; f_{i}=\frac{b_{i}}{\sum_{j=0}^{q} b_{j}}} \\
\alpha=[\mathrm{H}]-[\mathrm{OH}]=10^{-\mathrm{pH}}-10^{\mathrm{pH}-\mathrm{p} K_{\mathrm{W}}}
\end{gathered}
$$

and applying the formula for mean number of protons attached to $\mathrm{L}^{-n}$ [2]

$$
\bar{n}=\frac{\sum_{i=1}^{q} i \cdot\left[\mathrm{H}_{i} \mathrm{~L}\right]}{\sum_{i=0}^{q}\left[\mathrm{H}_{i} \mathrm{~L}\right]}=\frac{\sum_{i=0}^{q} i \cdot K_{i}^{\mathrm{H}} \cdot[\mathrm{H}]^{i}}{\sum_{j=0}^{q} K_{j}^{\mathrm{H}} \cdot[\mathrm{H}]^{j}}=\frac{\sum_{i=0}^{q} i \cdot b_{i}}{\sum_{j=0}^{q} b_{j}}=\sum_{i=0}^{q} i \cdot f_{i}=\sum_{i=1}^{q} i \cdot f_{i}
$$

in the charge balance equation

$$
\alpha+[\mathrm{M}]+[\mathrm{K}]+\sum_{i=0}^{q}(i-n)\left[\mathrm{H}_{i} \mathrm{~L}\right]=0
$$

we get, by turns,

$$
\begin{gathered}
\alpha+\frac{C \cdot V}{V_{0}+V}+m \cdot \frac{C_{0} \cdot V_{0}}{V_{0}+V}=(n-\bar{n}) \cdot \frac{C_{0} \cdot V_{0}}{V_{0}+V} \\
V=V_{0} \cdot \frac{(n-m-\bar{n}) \cdot C_{0}-\alpha}{C+\alpha} \\
V_{0}+V=V_{0} \cdot \frac{(n-m-\bar{n}) \cdot C_{0}+C}{C+\alpha} \\
=\left((n-m) \cdot C_{0}+C\right) \cdot V_{0} \cdot \frac{1}{C+\alpha}-C_{0} \cdot V_{0} \cdot \frac{\bar{n}}{C+\alpha}
\end{gathered}
$$

Differentiating Eq. 10 gives:

$$
\begin{aligned}
\frac{\mathrm{d}\left(V_{0}+V\right)}{\mathrm{dpH}} & =\frac{\mathrm{d} V}{\mathrm{dpH}} \\
& =-\left((n-m) C_{0}+C\right) \cdot V_{0} \cdot \frac{1}{(C+\alpha)^{2}} \cdot \frac{\mathrm{d} \alpha}{\mathrm{dpH}}-C_{0} \cdot V_{0} \cdot \frac{\frac{\mathrm{d} \bar{n}}{\mathrm{dpH}} \cdot(C+\alpha)-\bar{n} \cdot \frac{\mathrm{d} \alpha}{\mathrm{dpH}}}{(C+\alpha)^{2}}
\end{aligned}
$$

Applying the relation:

$$
\frac{\mathrm{d} z}{\mathrm{dpH}}=\frac{\mathrm{d} z}{\mathrm{~d}[\mathrm{H}]} \cdot \frac{\mathrm{d}[\mathrm{H}]}{\mathrm{dpH}}=-\ln 10 \cdot[\mathrm{H}] \cdot \frac{\mathrm{d} z}{\mathrm{~d}[\mathrm{H}]}
$$

for $\mathrm{z}=\alpha$ (Eq. 5) and $\bar{n}$ (Eq. 6), we get [2, 12]: 


$$
\begin{gathered}
\frac{\mathrm{d} \alpha}{\mathrm{dpH}}=-\ln 10 \cdot([\mathrm{H}]+[\mathrm{OH}]) \\
\frac{\mathrm{d} \bar{n}}{\mathrm{dpH}}=-\ln 10 \cdot \sum_{j>i=0}^{q}(j-i)^{2} \cdot f_{i} f_{j}
\end{gathered}
$$

and then from Eq. 11 we have:

$$
\begin{aligned}
\frac{\mathrm{d} V}{\mathrm{dpH}}= & \frac{V_{0} \cdot \ln 10}{(C+\alpha)^{2}} \cdot\left(\left((n-m) \cdot C_{0}+C-C_{0} \cdot \sum_{i=1}^{q} i \cdot f_{i}\right) \cdot([\mathrm{H}]+[\mathrm{OH}])\right. \\
& \left.+C_{0} \cdot(C+\alpha) \cdot \sum_{j>i=0}^{q}(j-i)^{2} \cdot f_{i} f_{j}\right)
\end{aligned}
$$

Note that $[\mathrm{H}]+[\mathrm{OH}]=\left(\alpha^{2}+4 K_{\mathrm{W}}\right)^{1 / 2}$ [12] (see Eq. 5), where $K_{\mathrm{W}}=[\mathrm{H}][\mathrm{OH}]$.

System 1B: When $V \mathrm{~mL}$ of $\mathrm{HB}\left(C, \mathrm{~mol} \cdot \mathrm{L}^{-1}\right)$ is added into $V_{0} \mathrm{~mL}$ of $\mathrm{K}_{m} \mathrm{H}_{n-m} \mathrm{~L}\left(C_{0}\right.$, $\left.\mathrm{mol} \cdot \mathrm{L}^{-1}\right)$, we have $[\mathrm{B}]=C V /\left(V_{0}+V\right)$. Then $C$ is replaced by $-C$ in the related formulas, and we have:

$$
\begin{aligned}
V & =V_{0} \cdot \frac{(\bar{n}+m-n) \cdot C_{0}+\alpha}{C-\alpha} \\
& =V_{0} \cdot \frac{-(\bar{n}+m-n) \cdot C_{0}-\alpha}{-C+\alpha}=V_{0} \cdot \frac{(n-m-\bar{n}) \cdot C_{0}-\alpha}{-(C-\alpha)}
\end{aligned}
$$

As we see, Eq. 16 can be obtained by setting $-C$ for $C$ in the related formula. Applying it to Eq. 15 , we get

$$
\begin{aligned}
\frac{\mathrm{d} V}{\mathrm{dpH}}= & \frac{V_{0} \cdot \ln 10}{(C-\alpha)^{2}} \cdot\left(\left((n-m) \cdot C_{0}-C-C_{0} \cdot \sum_{i=1}^{q} i \cdot f_{i}\right) \cdot([\mathrm{H}]+[\mathrm{OH}])\right. \\
& \left.-C_{0} \cdot(C-\alpha) \cdot \sum_{j>i=0}^{q}(j-i)^{2} \cdot f_{i} f_{j}\right)
\end{aligned}
$$

System 2A: $V \mathrm{~mL}$ of $C \mathrm{~mol} \cdot \mathrm{L}^{-1} \mathrm{MOH}$ is added into $V_{0} \mathrm{~mL}$ of the mixture: $\mathrm{K}_{m_{k}} \mathrm{H}_{n_{k}-m_{k}} \mathrm{~L}_{(k)}\left(C_{0 k} ; m_{k}=0, \ldots, n_{k} ; k=1, \ldots, \mathrm{P}\right) ; \mathrm{H}_{n_{k}+m_{k}} \mathrm{~L}_{(k)} \mathrm{B}_{m_{k}}\left(C_{0 k} ; m_{k}=0, \ldots, q_{k}-n_{k} ;\right.$ $k=\mathrm{P}+1, \ldots, \mathrm{Q}), \mathrm{HB}\left(C_{0 \mathrm{a}}\right)$ and $\mathrm{MOH}\left(C_{0 \mathrm{~b}}\right)$. Denoting $-n_{k}-$ charge of $\mathrm{L}_{(k)}^{-n_{k}}$, we have the charge balance equation:

$$
\alpha+[\mathrm{K}]+[\mathrm{M}]-[\mathrm{B}]+\sum_{k=1}^{Q} \sum_{j=0}^{q_{k}}\left(j-n_{k}\right)\left[\mathrm{H}_{j} \mathrm{~L}_{k}\right]=0
$$

where:

$$
\sum_{j=0}^{q_{k}}\left[\mathrm{H}_{j} \mathrm{~L}_{(k)}\right]=\frac{C_{0 k} V_{0}}{V_{0}+V}(k=1, \ldots, \mathrm{P}, \mathrm{P}+1, \ldots, \mathrm{Q}) ;[\mathrm{K}]=\frac{\sum_{k=1}^{P} m_{k} C_{0 k} V_{0}}{V_{0}+V}
$$




$$
\begin{gathered}
{[\mathrm{M}]=\frac{C V+C_{0 b} V_{0}}{V_{0}+V}} \\
{[\mathrm{~B}]=\frac{\sum_{k=P+1}^{Q} m_{k} C_{0 k} V_{0}+C_{0 a} V_{0}}{V_{0}+V}}
\end{gathered}
$$

The presence of strong acid $\mathrm{HB}\left(C_{0 \mathrm{a}}\right)$ and $\mathrm{MOH}\left(C_{0 \mathrm{~b}}\right)$ in the titrand $\mathrm{D}$ can be perceived as a kind of pre-assumed/intentional "mess" done in stoichiometric composition of the salts. Denoting: $\left[\mathrm{H}_{i} \mathrm{~L}_{(k)}\right]=K_{k i}^{\mathrm{H}} \cdot[\mathrm{H}]^{i} \cdot\left[\mathrm{L}_{(k)}\right] ; b_{k i}=K_{k i}^{\mathrm{H}} \cdot[\mathrm{H}]^{i}$, and

$$
f_{k i}=\frac{b_{k i}}{\sum_{j=0}^{q_{k}} b_{k j}} ; \quad \bar{n}_{k}=\frac{\sum_{i=0}^{q_{k}} i \cdot\left[\mathrm{H}_{i} \mathrm{~L}_{(k)}\right]}{\sum_{j=0}^{q_{k}}\left[\mathrm{H}_{j} \mathrm{~L}_{(k)}\right]}=\frac{\sum_{i=0}^{q_{k}} i K_{k i}^{\mathrm{H}} \cdot[\mathrm{H}]^{i}}{\sum_{j=0}^{q_{k}} K_{k j}^{\mathrm{H}} \cdot[\mathrm{H}]^{j}}=\frac{\sum_{i=0}^{q_{k}} i b_{k i}}{\sum_{j=0}^{q_{k}} b_{k j}}=\sum_{i=1}^{q_{k}} i \cdot f_{k i}
$$

we have:

$$
\frac{\mathrm{d} \bar{n}_{k}}{\mathrm{dpH}}=-\ln 10 \cdot \sum_{j>i=0}^{q_{k}}(j-i)^{2} \cdot f_{k i} f_{k j}
$$

Introducing Eqs. 19-23 into Eq. 18 we get, by turns:

$$
\begin{aligned}
& \alpha+\frac{\sum_{k=1}^{p} m_{k} C_{0 k} V_{0}}{V_{0}+V}+\frac{C V+C_{0 b} V_{0}}{V_{0}+V}-\frac{\sum_{k=p+1}^{Q} m_{k} C_{0 k} V_{0}+C_{0 a} V_{0}}{V_{0}+V}+\sum_{k=1}^{Q} \sum_{i=0}^{q_{k}}\left(i-n_{k}\right)\left[\mathrm{H}_{i} \mathrm{~L}_{(k)}\right]=0 \\
& \alpha V_{0}+\alpha V+\sum_{k=1}^{p} m_{k} C_{0 k} V_{0}+C V+\Delta_{0} V_{0}-\sum_{k=p+1}^{Q} m_{k} C_{0 k} V_{0}-\sum_{k=1}^{Q} n_{k} \cdot C_{0 k} V_{0} \\
& +\sum_{k=1}^{Q} \bar{n}_{k} \cdot C_{0 k} V_{0}=0 \\
& V_{0}+V=V_{0} \cdot\left(\sum_{k=1}^{P}\left(n_{k}-m_{k}\right) \cdot C_{0 k}+\sum_{k=P+1}^{Q}\left(n_{k}+m_{k}\right) \cdot C_{0 k}-\Delta_{0}+C\right) \cdot \frac{1}{C+\alpha} \\
& \quad-V_{0} \cdot \frac{\sum_{k=1}^{Q} \bar{n}_{k} \cdot C_{0 k}}{C+\alpha} \\
& \quad \frac{\mathrm{d} V}{\mathrm{dpH}}=\frac{V_{0} \cdot \ln 10}{(C+\alpha)^{2}} \cdot\left(\sum_{k=1}^{P}\left(n_{k}-m_{k}\right) \cdot C_{0 k}+\sum_{k=P+1}^{Q}\left(n_{k}+m_{k}\right) \cdot C_{0 k}\right. \\
& \left.\quad-\sum_{k=1}^{Q} C_{0 k} \cdot \sum_{i=1}^{q_{k}} i \cdot f_{k i}-\Delta_{0}+C\right) \cdot([H]+[O H]) \\
& \left.+\sum_{k=1}^{Q} C_{0 k} \cdot \sum_{j>i=0}^{q_{k}}(j-i)^{2} \cdot f_{k i} f_{k j}\right) \cdot
\end{aligned}
$$

where 


$$
\Delta_{0}=C_{0 b}-C_{0 a}
$$

System 2B: $V \mathrm{~mL}$ of $C \mathrm{~mol} \cdot \mathrm{L}^{-1} \mathrm{HB}$ is added into $V_{0} \mathrm{~mL}$ of the mixture: $\mathrm{K}_{m_{k}} \mathrm{H}_{n_{k}-m_{k}} \mathrm{~L}_{(k)}$ $\left(C_{0 k} ; m_{k}=0, \ldots, n_{k} ; k=1, \ldots, \mathrm{P}\right) ; \mathrm{H}_{n_{k}+m_{k}} \mathrm{~L}_{(k)} \mathrm{B}_{m_{k}}\left(C_{0 k} ; m_{k}=0, \ldots, q_{k}-n_{k} ; k=\mathrm{P}+1, \ldots, \mathrm{Q}\right)$, $\mathrm{HB}\left(C_{0 \mathrm{a}}\right)$ and $\mathrm{MOH}\left(C_{0 \mathrm{~b}}\right)$. We have the balances Eqs. 18 and 19, and

$$
\begin{gathered}
{[\mathrm{M}]=\frac{C_{0 b} V_{0}}{V_{0}+V}} \\
{[\mathrm{~B}]=\frac{\sum_{k=P+1}^{Q} m_{k} C_{0 k} V_{0}+C_{0 a} V_{0}+C V}{V_{0}+V}}
\end{gathered}
$$

Introducing Eqs. 19, 27, 28 into Eq. 18 and applying Eqs. 13, 22, 23, 26 we obtain:

$$
\begin{aligned}
\frac{\mathrm{d} V}{\mathrm{dpH}}= & \frac{V_{0} \cdot \ln 10}{(C-\alpha)^{2}} \cdot\left(\left(\sum_{k=1}^{P}\left(n_{k}-m_{k}\right) \cdot C_{0 k}+\sum_{k=P+1}^{Q}\left(n_{k}+m_{k}\right) \cdot C_{0 k}\right.\right. \\
& \left.-\Delta_{0}-C-\sum_{k=1}^{Q} C_{0 k} \cdot \sum_{i=1}^{q_{k}} i \cdot f_{k i} \cdot\right) \cdot([\mathrm{H}]+[\mathrm{OH}]) . . \\
& \left.-(C-\alpha) \cdot \sum_{k=1}^{Q} C_{0 k} \cdot \sum_{j>i=0}^{q_{k}}(j-i)^{2} \cdot f_{k i} f_{k j}\right)
\end{aligned}
$$

System 3A: $V \mathrm{~mL}$ of $C \mathrm{~mol} \cdot \mathrm{L}^{-1} \mathrm{M}_{m} \mathrm{H}_{n-m} \mathrm{~L}$ is added into $V_{0} \mathrm{~mL}$ of the mixture: $\mathrm{K}_{m_{k}} \mathrm{H}_{n_{k}-m_{k}} \mathrm{~L}_{(k)}\left(C_{0 k} ; m_{k}=0, \ldots, n_{k} ; k=1, \ldots, \mathrm{P}\right) ; \mathrm{H}_{n_{k}+m_{k}} \mathrm{~L}_{(k)} \mathrm{B}_{m_{k}}\left(C_{0 k} ; m_{k}=0, \ldots, q_{k}-n_{k} ;\right.$ $k=\mathrm{P}+1, \ldots, \mathrm{Q}), \mathrm{HB}\left(C_{0 \mathrm{a}}\right)$ and $\mathrm{MOH}\left(C_{0 \mathrm{~b}}\right)$. From charge

$$
\alpha+[\mathrm{K}]+[\mathrm{M}]-[\mathrm{B}]+\sum_{k=1}^{Q} \sum_{j=0}^{q_{k}}\left(j-n_{k}\right)\left[\mathrm{H}_{j} \mathrm{~L}_{(k)}\right]+\sum_{j=0}^{q}(j-n)\left[\mathrm{H}_{j} \mathrm{~L}\right]=0
$$

and concentration balances, Eqs. 19 and 21 and

$$
\begin{aligned}
& \sum_{j=0}^{q}\left[\mathrm{H}_{j} \mathrm{~L}\right]=\frac{C V}{V_{0}+V} \\
& {[\mathrm{M}]=\frac{m C V+C_{0 b} V_{0}}{V_{0}+V}}
\end{aligned}
$$

after introducing Eqs. 19, 21, 31, 32 into Eq. 30 and applying Eqs. 6, 13, 14, 22, 23 and 26, we obtain:

$$
\begin{aligned}
V_{0}+V= & V_{0} \cdot\left(-\sum_{k=1}^{P}\left(n_{k}-m_{k}\right) C_{0 k}-\sum_{k=P+1}^{Q}\left(n_{k}+m_{k}\right) C_{0 k}+(n-m) \cdot C+\Delta_{0}\right) . \\
& \frac{1}{(n-m-\bar{n}) \cdot C-\alpha}+V_{0} \cdot \frac{\sum_{k=1}^{Q} \bar{n}_{k} \cdot C_{0 k}-\bar{n} \cdot C}{(n-m-\bar{n}) \cdot C-\alpha}
\end{aligned}
$$

and then 


$$
\begin{aligned}
\frac{\mathrm{d} V}{\mathrm{dpH}}= & \frac{V_{0} \cdot \ln 10}{\left(\left(n-m-\sum_{i=1}^{q} i \cdot f_{i}\right) \cdot C-\alpha\right)^{2}} \cdot\left(\left(\sum_{k=1}^{P}\left(n_{k}-m_{k}\right) \cdot C_{0 k}+\sum_{k=P+1}^{Q}\left(n_{k}+m_{k}\right) \cdot C_{0 k}\right.\right. \\
& \left.-\sum_{k=1}^{Q} C_{0 k} \cdot \sum_{i=1}^{q_{k}} i \cdot f_{k i}+\left(\sum_{i=1}^{q} i \cdot f_{i}-n+m\right) \cdot C-\Delta_{0}\right) \\
& \cdot\left(C \cdot \sum_{j>i=0}^{q}(j-i)^{2} \cdot f_{i} f_{j}+[\mathrm{H}]+[\mathrm{OH}]\right)-\left(\left(n-m-\sum_{i=1}^{q} i \cdot f_{i}\right) \cdot C-\alpha\right) \\
& \left.\cdot\left(\sum_{k=1}^{Q} C_{0 k} \cdot \sum_{j>i=0}^{q_{k}}(j-i)^{2} \cdot f_{k i} f_{k j}-C \cdot \sum_{j>i=0}^{q}(j-i)^{2} \cdot f_{i} f_{j}\right)\right)
\end{aligned}
$$

System 3B: $V \mathrm{~mL}$ of $C \mathrm{~mol} \cdot \mathrm{L}^{-1} \mathrm{H}_{n+m} \mathrm{LB}_{m}$ is added into $V_{0} \mathrm{~mL}$ of the mixture: $\mathrm{K}_{m_{k}} \mathrm{H}_{n_{k}-m_{k}} \mathrm{~L}_{(k)}\left(C_{0 k} ; m_{k}=0, \ldots, n_{k} ; k=1, \ldots, \mathrm{P}\right) ; \mathrm{H}_{n_{k}+m_{k}} \mathrm{~L}_{(k)} \mathrm{B}_{m_{k}}\left(C_{0 k} ; m_{k}=0, \ldots, q_{k}-n_{k} ;\right.$ $k=\mathrm{P}+1, \ldots, \mathrm{Q}), \mathrm{HB}\left(C_{0 \mathrm{a}}\right)$ and $\mathrm{MOH}\left(C_{0 \mathrm{~b}}\right)$. Applying Eqs. $19,27,31$ and

$$
[\mathrm{B}]=\frac{\sum_{k=P+1}^{Q} m_{k} \cdot C_{0 k} V_{0}+C_{0 a} V_{0}+m \cdot C V}{V_{0}+V}
$$

in Eq. 30, we obtain:

$$
\begin{aligned}
& V_{0}+V=V_{0} \\
& \cdot \frac{-\sum_{k=1}^{P}\left(n_{k}-m_{k}\right) \cdot C_{0 k}-\sum_{k=P+1}^{Q}\left(n_{k}+m_{k}\right) \cdot C_{0 k}+\sum_{k=1}^{Q} \bar{n}_{k} \cdot C_{0 k}+\Delta_{0}+(n+m-\bar{n}) \cdot C}{(n+m-\bar{n}) \cdot C-\alpha} \\
& V_{0}+V=V_{0} \cdot\left(-\sum_{k=1}^{P}\left(n_{k}-m_{k}\right) \cdot C_{0 k}-\sum_{k=P+1}^{Q}\left(n_{k}+m_{k}\right) \cdot C_{0 k}+(n+m) \cdot C+\Delta_{0}\right) \\
& \cdot \frac{1}{(n+m-\bar{n}) \cdot C-\alpha}+V_{0} \cdot \frac{\sum_{k=1} \bar{n}_{k} \cdot C_{0 k}-\bar{n} \cdot C}{(n+m-\bar{n}) \cdot C-\alpha}
\end{aligned}
$$

Then applying Eqs. 6, 13, 14, 23 and 24 in 37, we have: 


$$
\begin{aligned}
\frac{\mathrm{d} V}{\mathrm{dpH}}= & \frac{V_{0} \cdot \ln 10}{\left(\left(n+m-\sum_{i=1}^{q} i \cdot f_{i}\right) \cdot C-\alpha\right)^{2}} \cdot\left(\sum_{k=1}^{P}\left(n_{k}-m_{k}\right) \cdot C_{0 k}+\sum_{k=P+1}^{Q}\left(n_{k}+m_{k}\right) \cdot C_{0 k}\right. \\
& \left.-(n+m) \cdot C-\Delta_{0}-\sum_{k=1}^{Q} C_{0 k} \cdot \sum_{i=1}^{q_{k}} i \cdot f_{k i}+C \cdot \sum_{i=1}^{q} i \cdot f_{i}\right) \\
& \cdot\left(C \cdot \sum_{j>i=0}^{q}(j-i)^{2} \cdot f_{i} f_{j}+[\mathrm{H}]+[\mathrm{OH}]\right) \\
& -\left(\sum_{k=1}^{Q} C_{0 k} \cdot \sum_{j>i=0}^{q_{k}}(j-i)^{2} \cdot f_{k i} f_{k j}-C \cdot \sum_{j>i=0}^{q}(j-i)^{2} \cdot f_{i} f_{j}\right) \\
& \times\left(\left(n+m-\sum_{i=1}^{q} i \cdot f_{i}\right) \cdot C-\alpha\right)
\end{aligned}
$$

In all cases it is assumed that $\beta_{V} \geq 0$; for this purpose, the absolute value (modulus) was introduced in Eq. 1. An analogous assumption was made for the static buffer capacity $(\beta)$.

\section{Britton-Robinson Buffers (BRB)}

Two buffers proposed by Britton and Robinson [14], marked as BRB-I and BRB-II, are obtained by titration to the desired $\mathrm{pH}$ value over the $\mathrm{pH}$ range 2-12 [15]. The $\mathrm{D}(V=10$ $\mathrm{mL})$ in BRB-I, consisting of $\mathrm{H}_{3} \mathrm{BO}_{3}\left(C_{01}\right)+\mathrm{H}_{3} \mathrm{PO}_{4}\left(C_{02}\right)+\mathrm{CH}_{3} \mathrm{COOH}\left(C_{03}\right)$, is titrated to the desired $\mathrm{pH}$ with $\mathrm{NaOH}(C)$ as $\mathrm{T}$; in this case, $C_{01}=C_{02}=C_{03}=0.04 \mathrm{~mol} \cdot \mathrm{L}^{-1}$, and $C=0.2 \mathrm{~mol} \cdot \mathrm{L}^{-1}$. The D in BRB-II, consisting of $\mathrm{H}_{3} \mathrm{BO}_{3}\left(C_{01}\right)+\mathrm{KH}_{2} \mathrm{PO}_{4}\left(C_{02}\right)+$ citric acid $\mathrm{H}_{3} \mathrm{~L}_{(3)}\left(C_{03}\right)+$ veronal $\mathrm{HL}_{(4)}+\mathrm{HCl}\left(C_{0 \mathrm{a}}\right)$, is titrated to the desired $\mathrm{pH}$ with $\mathrm{NaOH}(\mathrm{C})$ as $\mathrm{T}$; in this case $C_{01}=C_{02}=C_{03}=C_{04}=C_{0 \mathrm{a}}=0.0286 \mathrm{~mol} \cdot \mathrm{L}^{-1}$, and $C=0.2 \mathrm{~mol} \cdot \mathrm{L}^{-1}$. For BRB-I we have the equation for the titration curve:

$$
V=V_{0} \cdot \frac{\left(3-\bar{n}_{1}\right) \cdot C_{01}+\left(3-\bar{n}_{2}\right) \cdot C_{02}+\left(1-\bar{n}_{3}\right) \cdot C_{03}-\alpha}{C+\alpha}
$$

(see Fig. 1), where:

$$
\begin{aligned}
\bar{n}_{1}= & \left(3 \times 10^{34.24-3 \mathrm{pH}}+2 \times 10^{25.7-2 \mathrm{pH}}+10^{13.3-\mathrm{pH}}\right) /\left(10^{34.24-3 \cdot \mathrm{pH}}+10^{25.7-2 \mathrm{pH}}\right. \\
& \left.+10^{13.3-\mathrm{pH}}+1\right) \\
\bar{n}_{2}= & \left(3 \times 10^{21-71 \mathrm{pH}}+2 \times 10^{19.59-2 \mathrm{pH}}+10^{12.38-\mathrm{pH}}\right) /\left(10^{21.71-3 \mathrm{pH}}+10^{19.59-2 \mathrm{pH}}\right. \\
& \left.+10^{12.38-\mathrm{pH}}+1\right) \\
& \bar{n}_{3}=10^{4.76-\mathrm{pH}} /\left(10^{4.76-\mathrm{pH}}+1\right)
\end{aligned}
$$

For the BRB-II buffer we have the equation for titration curve 


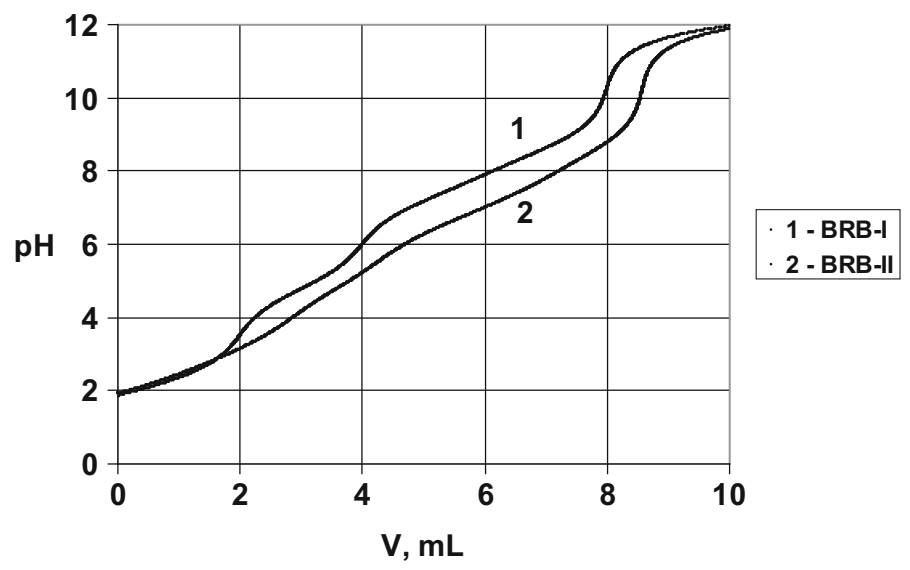

Fig. 1 Curves of titration of BRB-I and BRB-II with NaOH. For details see the text
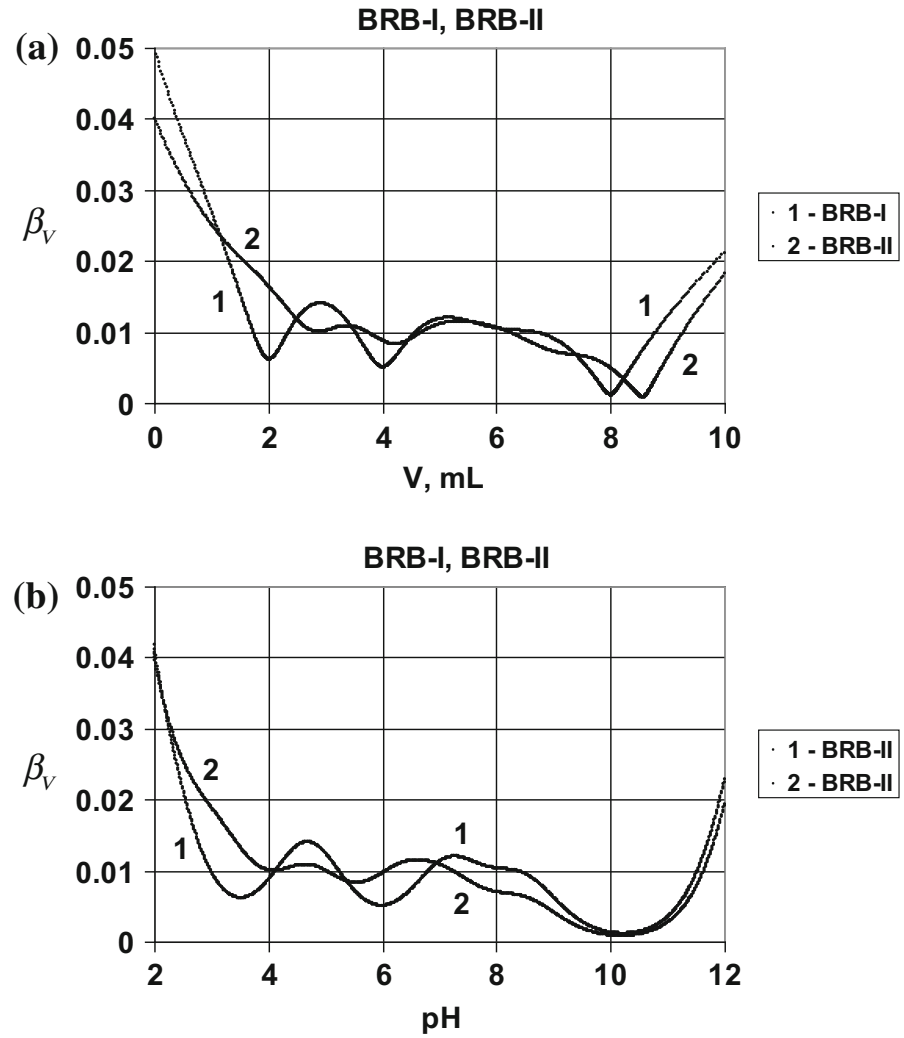

Fig. 2 The plots of $\mathbf{a} \beta_{V}$ vs. $V$ and $\mathbf{b} \beta_{V}$ vs. pH relationships obtained for BRB-I and BRB-II. For details see the text 


$$
V=V_{0} \cdot \frac{\left(3-\bar{n}_{1}\right) \cdot C_{01}+\left(2-\bar{n}_{2}\right) \cdot C_{02}+\left(3-\bar{n}_{3}^{\bullet}\right) \cdot C_{03}+\left(1-\bar{n}_{4}\right) \cdot C_{04}+C_{0 a}-\alpha}{C+\alpha}
$$

(see Figs. 1, 2), where $\bar{n}_{1}($ Eq. 40$)$ and $\bar{n}_{2}$ (Eq. 41) and:

$$
\begin{gathered}
\bar{n}_{3}^{\bullet}=\left(3 \times 10^{14.28-3 \mathrm{pH}}+2 \times 10^{11.15-2 \mathrm{pH}}+10^{6.39-\mathrm{pH}}\right) /\left(10^{14.28-3 \mathrm{pH}}+10^{11.15-2 \cdot \mathrm{pH}}\right. \\
\left.+10^{6.39-\mathrm{pH}}+1\right) \\
\bar{n}_{4}=10^{7.43-\mathrm{pH}} /\left(10^{7.43-\mathrm{pH}}+1\right)
\end{gathered}
$$

The formulas for $\bar{n}_{i}(i=1, \ldots, 4)$ and $\bar{n}_{3}^{\bullet}$ in Eqs. 39 and 43 were obtained on the basis of $\mathrm{p} K_{i}$ values found in [16-20].

Note that

$$
\begin{aligned}
& \sum_{j>i=0}^{3}(j-i)^{2} \cdot f_{k i} f_{k j}=f_{k 1} f_{k 0}+4 f_{k 2} f_{k 0}+9 f_{k 3} f_{k 0}+f_{k 2} f_{k 1}+4 f_{k 3} f_{k 1}+f_{k 3} f_{k 2} ; \\
& \sum_{j>i=0}^{2}(j-i)^{2} \cdot f_{k i} f_{k j}=f_{k 1} f_{k 0}+4 f_{k 2} f_{k 0}+f_{k 2} f_{k 1} ; \sum_{j>i=0}^{1}(j-i)^{2} \cdot f_{k i} f_{k j}=f_{k 1} f_{k 0} .
\end{aligned}
$$

\section{Final Comments}

The mathematical formulation of the dynamic buffer capacity $\beta_{V}$ concept is presented in a general and elegant form, involving all soluble species formed in the system where only acid-base reactions are involved. This approach to buffer capacity is more general than one presented in the earlier study [2] and is correct from a mathematical viewpoint, in contrast to the one presented in [21]. It is also an extension of an earlier approach, presented for less complex acid-base static [8] and dynamic [10, 12] systems. The calculations were exemplified with two complex buffers, proposed by Britton and Robinson [14].

The salts specified in particular systems considered above do not cover all possible types of the salts, e.g. $\left(\mathrm{NH}_{4}\right)_{2} \mathrm{HPO}_{4}$ or potassium sodium tartrate $(\mathrm{KNaL})$ are not examples of the salts of $\mathrm{K}_{m_{k}} \mathrm{H}_{n_{k}-m_{k}} \mathrm{~L}_{(k)}$ or $\mathrm{H}_{n_{k}+m_{k}} \mathrm{~L}_{(k)} \mathrm{B}_{m_{k}}$ type. However, in $\mathrm{D},\left(\mathrm{NH}_{4}\right)_{2} \mathrm{HPO}_{4}\left(C_{0 i}\right)$ is equivalent to a mixture of $\mathrm{NH}_{3}\left(2 C_{0 i}\right)$ and $\mathrm{H}_{3} \mathrm{PO}_{4}\left(C_{0 i}\right)$, whereas $\mathrm{KNaL}\left(C_{0 j}\right)$ is equivalent to a mixture of $\mathrm{NaOH}\left(C_{0 j}\right), \mathrm{KOH}\left(C_{0 j}\right)$ and $\mathrm{H}_{2} \mathrm{~L}\left(C_{0 j}\right)$.

Open Access This article is distributed under the terms of the Creative Commons Attribution 4.0 International License (http://creativecommons.org/licenses/by/4.0/), which permits unrestricted use, distribution, and reproduction in any medium, provided you give appropriate credit to the original author(s) and the source, provide a link to the Creative Commons license, and indicate if changes were made.

\section{References}

1. Albert, A., Serjeant, E.P.: The Determination of Ionisation Constants. Chapman and Hall, London (1984)

2. Asuero, A.G., Michałowski, T.: Comprehensive formulation of titration curves referred to complex acid-base systems and its analytical implications. Crit. Rev. Anal. Chem. 41, 151-187 (2011) 
3. He, J.-L., Li, H.-P., Li, X.-G.: Analysis of prostaglandins in SD rats by capillary zone electrophoresis with undirected UV detection. Talanta 46, 1-7 (1998)

4. Schneede, J., Ueland, P.M.: The formation in an aqueous matrix, properties and chromatographic behavior of 1-pyrenyldiazomethane derivatives of methylmalonic acid and other short chain dicarboxylic acids. Anal. Chem. 64, 315-319 (1992)

5. Lagane, B., Treilhou, M., Couderc, F.: Capillary electrophoresis: theory, teaching approach and separation of oligosaccharides using indirect UV detection. Biochem. Mol. Biol. Educ. 28, 251-255 (2000). http://www.sciencedirect.com/science/article/pii/S147081750000031X

6. Jordan, C.: Ionic strength and buffer capacity of wide-range buffers for polarography. Microchem. J. 25, 492-499 (1980)

7. Van Slyke, D.D.: On the measurement of buffer values and on the relationship of buffer value to the dissociation constant of the buffer and the concentration and reaction of the buffer solution. J. Biol. Chem. 52, 525-570 (1922). http://www.jbc.org/content/52/2/525.full.pdf + html. Accessed 24 May 2015

8. Hesse, R., Olin, A.: A simple expression for the buffer index of a weak polyprotic acid. Talanta 24, 150 (1977)

9. Butler, J.N.: Solubility and pH Calculations. Addison-Wesley Publishing Company Inc., Reading Mass (1964)

10. Michałowski, T., Parczewski, A.: A new definition of buffer capacity. Chem. Anal. 23, 959-964 (1978)

11. Moisio, T., Heikonen, M.: A simple method for the titration of multicomponent acid-base mixtures. Fresenius' J. Anal. Chem. 354, 271-277 (1996)

12. Michalowski, T.: Some remarks on acid-base titration curves. Chem. Anal. 26, 799-813 (1981)

13. Asuero, A.G., Jiménez-Trillo, J.L., Navas, M.J.: Mathematical treatment of absorbance versus pH graphs of polybasic acids. Talanta 33, 929-934 (1986)

14. Britton, H.T.K., Robinson, R.A.: Universal buffer solutions and the dissociation constant of veronal. J. Chem. Soc. 10, 1456-1462 (1931). http://www.oalib.com/references/13396293

15. http://en.wikipedia.org/wiki/Britton-Robinson_buffer. Accessed 24 May 2015

16. http://en.wikipedia.org/wiki/Boric_acid. Accessed 24 May 2015

17. http://en.wikipedia.org/wiki/Phosphoric_acid. Accessed 24 May 2015

18. http://en.wikipedia.org/wiki/Citric_acid. Accessed 24 May 2015

19. http://en.wikipedia.org/wiki/Acetic_acid. Accessed 24 May 2015

20. http://www.zirchrom.com/organic.htm. Accessed 24 May 2015

21. Rojas-Hernández, A., Rodríguez-Laguna, N., Ramírez-Silva, M.T., Moya-Hernández, R.: Distribution diagrams and graphical methods to determine or to use the stoichiometric coefficients of acid-base and complexation reactions. In: Innocenti, A. (ed.) Stoichiometry and Research-The Importance of Quantity in Biomedicine, InTech, Rijeka, Croatia, pp. 287-310. (2012). http://www.intechopen.com/ books/stoichiometry-and-research-the-importance-of-quantity-in-biomedicine 